\title{
Über den Einfluß der Temperatur auf biologische Strahlenwirkungen
}

\author{
2. Mitt.: Inaktivierung von trockenen und feuchten Sporen von Baz. subtilis \\ durch $a-$ und Röntgenstrahlen \\ Von Thea Houtermans
}

\author{
Aus dem Hygiene-Institut der Universität Göttingen \\ (Z. Naturforschg. 11 b, 636-643 [1956]; eingegangen am 19. Juni 1956)
}

\begin{abstract}
Der Einfluß der Temperatur auf die $\alpha$ - und Röntgenstrahlen-Inaktivierung von trockenen und feuchten Sporen von B. subtilis wurde für das Temperaturintervall von $-184^{\circ} \mathrm{C}$ bis $+20^{\circ} \mathrm{C}$ gemessen. Für trockene Sporen waren die Inaktivierungskurven in Abhängigkeit von der Temperatur für Röntgen- und $\alpha$-Strahlen ähnlich. Für beide Strahlenarten nahm die Inaktivierungs-Wahrscheinlichkeit unterhalb von $-30^{\circ} \mathrm{C}$ ab. Der Einfluß der Feuchtigkeit war für $\alpha$ - und Röntgenstrahlen verschieden. Gegenüber Röntgenstrahlen waren die feuchten Sporen unempfindlicher als die trockenen, und die Inaktivierungs-Wahrscheinlichkeit zeigte ebenfalls einen Abfall unterhalb von $-30^{\circ} \mathrm{C}$ und erreichte bei sehr tiefen Temperaturen praktisch den für trockene Sporen gemessenen Wert. Gegenüber $\alpha$-Strahlen war die InaktivierungsWahrscheinlichkeit für feuchte und trockene Sporen bei $20^{\circ} \mathrm{C}$ praktisch gleich und blieb für feuchte Sporen im gesamten Temperaturintervall bis $-184^{\circ} \mathrm{C}$ nahezu konstant.
\end{abstract}

$\mathrm{A}^{\mathrm{u}}$ frund eines umfangreichen radiochemischen Versuchsmaterials wird allgemein als gut fundiert anerkannt, daß oft die Effekte ionisierender Strahlen auf chemische Substanzen in wäßriger Lösung im wesentlichen durch die bei der Absorption der Strahlung im Wasser erzeugten Radikale und deren Folgeprodukte hervorgerufen werden. Da lebende Zellen im allgemeinen einen hohen Prozentsatz Wasser enthalten, ist die Frage, in welchem Maße Zersetzungsprodukte des Wassers für die bei biologischen Objekten beobachteten Strahlenschäden verantwortlich sind, Ausgangspunkt zahlreicher biologischer Untersuchungen geworden*. Unter diesem Aspekt stehen auch die Versuche über die Abhängigkeit der Strahlenempfindlichkeit von der Temperatur und dem Wassergehalt während der Bestrahlung.

Die zunächst angenommene Temperaturunabhängigkeit der Strahlenwirkung schien mit Hypothesen, welche die Diffusion von Molekülen oder Radikalen einschließen, nicht in Einklang zu stehen. In neueren Untersuchungen wurde das untersuchte Temperaturintervall gegenüber früheren Messungen erweitert und erhebliche Temperaturabhängigkeiten gefunden. Meist wurde der Effekt bei Zimmertemperatur

* Die Begriffe „direkter“ und ,indirekter“ Effekt werden in dieser Arbeit bewußt nicht verwendet. Man hat bei der Durchsicht der einschlägigen Arbeiten nicht den Eindruck, daß eine bestimmte, klare Abgrenzung dieser beiden Begriffe gegeneinander allgemein anerkannt ist.

1 B. R a j e w s ky, Brit. J. Radiol. 25, 550 [1952].

2 A. C. F a bergé, Rad. Res. 1, 130 [1954].

3 L. H. Gray, Rad. Res. 1, 189 [1954].

mit dem bei einer sehr niedrigen Temperatur verglichen (z. B. R a je w sky ${ }^{1}$, F a be r g é ${ }^{2}$ ). Das Vorliegen einer geringeren Sensibilität bei niedrigen Temperaturen wurde als Hinweis auf eine maßgebliche Rolle der Wasserradikale angesehen (s. hierzu auch Gray ${ }^{3}$ ).

Die Berechtigung zu einer solchen Schlußfolgerung ist jedoch sehr in Frage gestellt durch zahlreiche Untersuchungen an trockenen organischen Substanzen (s. dazu Simoni s ${ }^{4}$ ), für die ebenfalls häufig eine solche Abhängigkeit von der Temperatur gemessen wurde (z. B. A d a m s und Polla r d ${ }^{5}$ für Invertase, Pollard ${ }^{6}$ für Proteine, Setlow und Doyle ${ }^{7}$ für Katalase). Auf Grund dieser Befunde kann das Vorliegen einer Temperaturabhängigkeit schlechthin nicht mehr als Hinweis auf Diffusionsvorgänge dienen. Dieser Schluß scheint jedoch dann naheliegend, wenn eine Unstetigkeit der Sensibilität beim Übergang vom festen in den flüssigen Zustand vorliegt. Dies ist jedoch nur wenig untersucht worden. S t a pleton und Eding ton ${ }^{8}$ haben mitgeteilt, daß sie für die Röntgenstrahlen-Inaktivierung von E. coli in $\mathrm{O}_{2}$-gesättigtem Milieu eine Diskontinuität beim Übergang vom flüssigen in den festen Zustand beob-

4 W. S im o n is, Fortschr. Bot. 17, 413 [1955].

5 W. R. A d a m s u. E. P o 11 a rd, Arch. Biochem. Biophysics 36, 311 [1952].

6 E. P o 11 a r d, Adv. Biol. Med. Phys. 3, 153 [1953]

7 R. S et low u. B. Do y le, Arch. Biochem. Biophysics 46, 46 [1953].

8 G. E. Stapleton u. C. W. Edington, Rad. Res. 1, 229 [1954]. 
achteten. Dies steht in gewissem Gegensatz zu eigenen, in einer 1. Mitt. ${ }^{9}$ beschriebenen Versuchen mit E. coli $\mathrm{H}$ ig, die in normaler Luftatmosphäre durchgeführt wurden. Eine sprunghafte Änderung der Strahlenempfindlichkeit beim Phasenwechsel trat nur bei $\alpha$-Bestrahlung auf, für die also ein Diffusionsmodell diskutiert werden kann. Bei Röntgenbestrahlung dagegen blieb die Empfindlichkeit unabhängig vom Aggregatzustand mindestens bis herunter $\mathrm{zu}$ $-10^{\circ} \mathrm{C}$ konstant und begann erst unterhalb dieser Temperatur abzunehmen. Ähnliche Verhältnisse fand W o o d ${ }^{10}$ für die Inaktivierung von Hefezellen durch Röntgenstrahlen. Er fand für Suspensionen in festem Zustand von $-10^{\circ} \mathrm{C}$ bis $0^{\circ} \mathrm{C}$ einen Anstieg der Sensibilität auf den für flüssige Suspensionen gemessenen Wert und sieht darin eine Stütze für ein Diffusionsmodell. Selbst wenn man wie Wood annimmt, daß bei Temperaturen, die nur wenig unter dem Gefrierpunkt liegen, noch ein geringer Teil des Wassers in "freier" Form vorliegt, erscheint doch die völlige Unabhängigkeit des Effektes bei $0^{\circ} \mathrm{C}$ vom Aggregatzustand der Suspension nur erklärbar, wenn man annimmt, daß im Inneren der Zelle infolge von Gefrierpunktserniedrigung noch kein fester Aggregatzustand vorliegt. Diese Erklärung kann jedoch kaum für die erwähnten eigenen Versuche mit E. coli gelten, da eine Gefrierpunktserniedrigung von $10^{\circ} \mathrm{C}$ nicht zu erwarten ist.

Eine andere Untersuchungsmethode, von der man sich Aufschluß über die Wirksamkeit der innerhalb der Zelle erzeugten Wasserradikale erhoffte, ist die Untersuchung der Abhängigkeit des Strahleneffektes vom Wassergehalt der Zellen. P i g m a n u. a. ${ }^{11,12}$ sowie $\mathrm{S}$ tapleton und $\mathrm{M}$ artin (zitiert nach $\mathrm{Hol}$ l a e n de r ${ }^{13}$ ) fanden für trockene Sporen von Aspergillus terreus eine geringere Röntgeninaktivierung als für Sporen mit höherem Wassergehalt. Auch Röntgenbestrahlungen von trockenen und gequollenen Samen deuteten zunächst in diese Richtung (z. B. K a plan ${ }^{14}$, Nybom u. a. ${ }^{15}$, Konzak ${ }^{16}$ ). Eingehendere Untersuchungen lieferten jedoch ein wesentlich komplexeres Bild. So erwiesen sich in Experimenten

\footnotetext{
9 T. Houtermans, Z. Naturforschg. 9 b, 600 [1954]

10 T. H. W o o d, Rad. Res. 1, 234 [1954].

11 W. Pig man, Jr. H. C. Elli ot u. R. O. L a f $\mathrm{fre}$, J. Dental Res. 31, 627 [1952].

12 W. Pigman, W. Hawkins u. A. Thom as, New York State Dental J. 22, 355 [1954].

13 A. Holl a e nder, Symp. Radiobiol. John Willy and Sons, Inc., New York 1952.

14 R. W. K a p l a n, Z. indukt. Abstammungs- u. Vererbungslehre 83, 347 [1951].
}

von $\mathrm{Calde} \operatorname{cott}{ }^{17}$ auch die gewässerten und wieder auf ihr Ausgangsgewicht getrockneten Samen als empfindlicher. Ein solcher Befund ist nicht erstaunlich, da bei der Samenquellung nicht nur der Wassergehalt geändert wird, sondern auch Keimungsprozesse eingeleitet werden. $\mathrm{C}$ a l de c o t $\mathrm{t}^{18}$ sowie $\mathrm{E} \mathrm{h}$ re n ber g ${ }^{19}$ fanden, daß eine Erhöhung der Strahlenempfindlichkeit erst bei einem Wassergehalt von über $20 \%$ auftritt. Im Bereich von 0 bis $20 \%$ dagegen wurde im Gegensatz zu den durch die Annahme einer Schädigung durch Wasserradikale implizierten Erwartungen eine Empfindlichkeits-Abnahme mit steigendem Wassergehalt beobachtet. Gerade in diesem Bereich aber ist es am wahrscheinlichsten, daß der Effekt durch die Anwesenheit von Wasser an sich bedingt ist, da die Empfindlichkeitsabnahme durch Trocknen wieder rückgängig gemacht werden konnte. Daß Wasser per se eine derartige „Schutzwirkung“ ausüben kann, zeigte E h r e n b e r g ${ }^{19}$ an Versuchen über die Spaltung von Kartoffelstärke durch Röntgenstrahlen, für die er ebenfalls ein Empfindlichkeitsminimum bei $20 \%$ Feuchtigkeit feststellte.

In der vorliegenden Arbeit ist der Einfluß der Temperatur auf die Strahleninaktivierung von Bazillus subtilis in feuchtem und trockenem Zustand untersucht worden.

Noch vor Abschluß der hier berichteten Experimente erschien eine Veröffentlichung von Proctor u. a. ${ }^{20}$ über Untersuchungen der Empfindlichkeit von Sporen von Baz. subtilis gegenüber Kathodenstrahlen bei verschiedenen Bedingungen, wobei auch Temperatur und Feuchtigkeitsgrad variiert wurden. Diese Ergebnisse sollen erst in der Diskussion am Schluß dieser Arbeit besprochen werden.

In Bazillensporen werden durch Wasser allein keine Entwicklungsprozesse eingeleitet. Noch immer ist die Ansicht weit verbreitet, daß Sporen sehr viel weniger Wasser enthalten als vegetative Bakterien. Eine Durchsicht der einschlägigen Literatur vermittelt jedoch den Eindruck, daß sich diese Behauptung lediglich auf den Umstand stützt, daß ein Wassergehalt von nur 10 bis $15 \%$ die hohe Hitzebeständig-

15 N. Nybom, A. Gustavson u. L. Ehrenb e r g, Bot. Notiser 4, 343 [1952].

16 C. F. K o n z a k, Rad. Res. 1, 220 [1954].

17 R. S. Caldecott, Rad. Res. 1, 490 [1954].

18 R. S. C a ld e c o t t, Science [Washington] 120, 809 [1954].

${ }_{19}$ L. E h re n berg, Vortrag, International Radiobiological Conference, Cambridge 1955.

20 B. E. Proctor, S. A. Goldblith, E. M. Oberle u. Jr. W. C. Miller, Rad. Res. 3, 295 [1955]. 
keit von Sporen sehr gut erklären würde (s. dazu auch $\mathrm{Henry}$ und Friedman ${ }^{21}$ ). Demgegenüber steht jedoch die Tatsache, daß alle bisherigen analytischen Untersuchungen zu dem Resultat führten, daß bei gleichen Milieubedingungen Sporen ebensoviel Wasser enthalten wie vegetative Zellen (Dyrmon t ${ }^{22}$, Virtanen und Pulk i i ${ }^{23}$, Hen ry und $\mathrm{Fr}$ i e d m a n ${ }^{21}$ ). Diese Ansicht wird inzwischen auch in der bakteriologischen Literatur weitgehend akzeptiert ( $\mathrm{L}$ a $\mathrm{m}$ a n n a ${ }^{24}$, W in k le r ${ }^{25}$ ).

\section{Material und Methoden}

\section{Biologisches Versuchsmaterial}

Weizengrieß-Agarplatten wurden mit vegetativen Keimen von Baz. subtilis (Stamm B aus der Sammlung des Hygiene-Institutes Göttingen) beimpft und bei $37^{\circ} \mathrm{C}$ bebrütet. Nach 6 Tagen waren die darauf gewachsenen Kulturen quantitativ versport. Sie wurden mit physiologischer Kochsalzlösung abgeschwemmt und mehrere Male, zunächst in Salzlösung, dann in dest. Wasser, gewaschen. Das Auswaschen in dest. Wasser wurde nach einigen Tagen wiederholt. Die so erhaltene wäßrige Sporen-Aufschwemmung wurde bei $4^{\circ} \mathrm{C}$ aufbewahrt. Sie diente als Ausgangsmaterial für alle hier aufgeführten Versuche, die etwa 14 Tage nach der letzten Auswaschung begannen.

Von auf Bouillonagar übertragenen Sporen keimten mehr als 99\% aus und entwickelten sich zu Kolonien. Noch nach einem Jahr war keinerlei Veränderung an den Sporen zu bemerken.

Es soll hier nochmals betont werden, daß die Milieubedingungen während der Bestrahlung keine „vorvegetativen" Veränderungen (s. darüber bei W in kle r ${ }^{25}$ ) bewirkten und es sich unter allen Versuchsbedingungen um ruhende Sporen handelte.

Versuchsanordnung für die Bestrah-

lung mit $50-k V-R o ̈ n t g e n s t r a h l e n$ einer

$\mathrm{S}$ pezial-Hochleist ung s anlage

Einige Versuche wurden mit einer Spezial-Hochleistungs-Röntgenanlage des biophysikalischen Institutes in Frankfurt a. M. durchgeführt. Eine Beschreibung der Anlage findet sich bei $\mathrm{Heuse}{ }^{26}$. Das Austrittsfenster für die Strahlung besteht aus drei Schichten von $1,5 \mathrm{~mm}$ Beryllium, $1 \mathrm{~cm}$ Wasser und $0,5 \mathrm{~mm}$ Beryllium. In $8 \mathrm{~mm}$ Fokusabstand bei einer Dosisleistung von $800 \mathrm{kr} / \mathrm{min}$ beträgt die Halbwertsschicht für Wasser $8 \mathrm{~mm}$. Diese Angaben vermitteln eine Vorstellung von der Härte der Strahlung. Bei den hier beschriebenen Versuchen betrug die Dosisleistung $200 \mathrm{kr} /$ Minute.

Zur Bestrahlung wurde aus der in dest. Wasser verdünnten Sporenemulsion (Dichte ca. $10^{7}$ Sporen $/ \mathrm{cm}^{3}$ ) $0,4 \mathrm{~cm}^{3}$ in Aluminiumschalen mit planem Boden pipettiert (Durchmesser der Bodenfläche 1,2 cm). Für die Be-

21 B. S. H e n ry u. C. A. F rie d m a n, J. Bacteriol. 33, 323 [1937].

22 A. D y rmo n t, Naunyn-Schmiedebergs Arch. exp. Pathol. Pharmakol. 21, 309 [1886].

23 A. J. Virtan en u. L. Pulkki, Arch. Mikrobiol. 4, 99 [1933]. strahlungen von trockenen Sporen wurden drei bis vier Stdn. vor Versuchsbeginn mit Hilfe einer Kapillare aus der Sporenemulsion Tröpfchen reproduzierbarer Masse (etwa $3 \mathrm{~mm}^{3}$ ) auf Glasplättchen oder Styroflexfolien gebracht, die bis zur Bestrahlung an der Luft trockneten. Anschließend wurden sie mit einer $200 \mu$ dicken Styroflexfolie bedeckt und in die oben beschriebenen Aluminiumschalen gesetzt, auf deren Boden ein winziger Wassertropfen gebracht war, um einen guten Temperaturausgleich zu gewährleisten. Zur Bestrahlung diente dieselbe Anordnung wie in den früheren Versuchen mit E. coli ${ }^{9}$. Die trockenen Sporen wurden nach der Bestrahlung sorgfältig mit einer Platinöse in etwas Wasser verrieben und schließlich in $1 \mathrm{~cm}^{3}$ Wasser abgeschwemmt. Aus den Emulsionen bestrahlter Sporen wurden dann nach geeigneter Verdünnung $0,05 \mathrm{~cm}^{3}$ in Petrischalen pipettiert und mit $15 \mathrm{~cm}^{3}$ Bouillonagar von $45^{\circ} \mathrm{C}$ vermischt. Die Bebrütung erfolgte bei $37^{\circ} \mathrm{C}$. Nach 24 Stdn. wurden die Kolonien ausgezählt.

Versuchsanordnung für die Bestrahlung mit dem Dermopan-Röntgengerät

Eingehendere Messungen, bei denen die Temperatur kontinuierlich von $+20^{\circ} \mathrm{C}$ bis $-184^{\circ} \mathrm{C}$ variiert wurde, wurden mit dem Dermopan-Röntgengerät der SiemensReiniger-Werke Erlangen durchgeführt. Dieses hat ein Beryllium-Fenster und liefert eine ungefilterte Strahlung sehr hoher Dosisleistung, die im wesentlichen durch den großen Anteil an sehr weicher Strahlung bedingt ist. Bei den Versuchen wurde mit einer Betriebsspannung von $50 \mathrm{kV}$ und einer Strombelastung von $25 \mathrm{~mA}$ gearbeitet.

Da die weiche Strahlung bereits in dünnen Schichten stark absorbiert wird, sind Vergleiche der Strahlenempfindlichkeit verschiedener Objekte nur dann exakt möglich, wenn die Bestrahlung bei derselben geometrischen Anordnung erfolgt. Die als „feucht“ bezeichneten Sporen waren daher auf feuchten Leeragarstückchen (Phosphatpuffer + Agar-Trockensubstanz) fixiert, auf die sie erst unmittelbar vor der Bestrahlung pipettiert wurden. Die trockenen Sporen waren wie bei den vorigen Versuchen auf Glas angetrocknet und in den meisten Fällen 24 Stdn. im Exsikkator aufbewahrt worden. Einige Versuche, bei denen die Sporen erst kurz vor der Bestrahlung getrocknet wurden, führten zu den gleichen Ergebnissen.

Abb. 1 zeigt einen Schnitt, Abb. 2 eine Aufsicht der Bestrahlungs-Apparatur. In ein Dewargefäß ist ein Kupferblock eingepaßt. Außerhalb des Dewargefäßes ist das Kupfer durch Kunststoff (Polyäthylen) gegen die Außenatmosphäre isoliert. Durch die Kunststoff-Deckplatte führen zwei Bohrungen in den Kupferblock, die beide in eine Höhlung münden. Über den Bohrungen sind Ansatzstutzen für Gummischläuche angebracht. Der Kupferblock kann auf beliebige Temperaturen zwischen Zimmertemperatur und $-184^{\circ} \mathrm{C}$ gebracht werden, indem man durch die Zuleitung flüssigen Sauerstoff einleitet, der in dem Kupferblock verdampft. Die Temperatur wird mittels eines Thermoelementes gemessen, dessen eine

24 C. L a m a n n a, Bacteriol. Rev. 16, 90 [1952].

25 A. W i n k l e r, Die Bakterienzelle. Gustav Fischer Verlag, Stuttgart 1956.

26 O. H e u s e, Z. angew. Physik 5, 361 [1953]. 
Lötstelle in das Kupfer eingelassen ist. Wenn in der Höhlung aller Sauerstoff verdampft ist, stellt sich nach ein bis zwei Min. ein Temperaturwert ein, der sich lange konstant hält. Die Wiedererwärmung erfolgt so langsam, daß sie für die kurzen Röntgen-Bestrahlungszeiten keine Rolle spielt. Selbstverständlich läßt sich bei einiger Übung durch vorsichtige Regulierung der Sauerstoffzufuhr die Temperatur über beliebig lange Zeit praktisch konstant halten.

In die Deckplatte ist ferner ein rundes Fenster eingeschnitten, das mit $10 \mu$ dickem Styroflex abgedeckt ist. Unmittelbar über dem Kupfer ist ein Schieber eingefügt mit zwei Ausschnitten für die zu bestrahlenden Präparate. Bei einer wie in Abb. 1 und 2 skizzierten Stellung des Schiebers wird das Präparat in den rechten Ausschnitt gelegt, wo es direkt auf dem Kupferblock liegt. Zwischen Sporen und Kupfer liegt eine dünne Schicht wärmeleitender Stoffe; gegen die Außentemperatur dagegen sind die Sporen durch schlechte Wärmeleiter isoliert. Sie nehmen daher praktisch die Temperatur des Kupfers an, die vom Thermoelement gemessen wird, und das gesamte Wärmegefälle vom Kupfer zur Außenatmosphäre liegt in der Luft- und Kunststoffschicht oberhalb der Sporen. Sicherheitshalber wurde dies durch Messungen mit einem zweiten, sehr kleinen Thermoelement geprüft. Nachdem der Temperaturangleich des Präparates erfolgt ist, wird es mittels des Schiebers zur Bestrahlung unter das Fenster geschoben.

Um das bei der Abkühlung durch die normale Luftfeuchtigkeit bedingte Beschlagen und Vereisen der Apparatur zu verhindern, steht sie in einer nahezu luftdichten Dry-box, deren Deckfläche aus Glas ist. Mindestens 24 Stdn. vor Versuchsbeginn wurden Schalen mit Phosphorpentoxyd-Pulver hineingesetzt und ein Ventilator in Betrieb genommen. Für den. Eintritt der Röntgenstrahlen ist ein Fenster aus $10 \mu$ dickem Stryroflex in die Deckglasplatte eingelassen. Behälter für flüssige Luft und das Meßinstrument für den Thermostrom befinden sich außerhalb der Dry-box. Die Schläuche für die Zuleitung des flüssigen und die Ableitung des verdampften Sauerstoffs und die Stromleitungen sind durch die Wand eingeführt. In die Vorderwand eingesetzte Gummihandschuhe ermöglichen das Manipulieren innerhalb der Dry-box. Die Präparate wurden durch eine Schleuse ein- und ausgeführt.

Von den bestrahlten Präparaten wurden die Sporen abgeschwemmt und in Agar gegossen, wie oben beschrieben. Daneben wurden jedoch auch mikroskopische Auswertungen vorgenommen (s. hierzu Engelhard und $\mathrm{Houtermans}{ }^{27}, \mathrm{H}$ outermans${ }^{28}$,, die zu gleichen Ergebnissen führten. Hinsichtlich beider Methoden erwiesen sich die hier verwandten Sporen als wesentlich unbequemeres Versuchsmaterial als etwa E.coli $\mathrm{H}$ i g. Gelegentliches Zusammenklumpen oder nicht vollständiges Abschwemmen der angetrockneten Sporen können bei der Plattenmethode zu Fehlern führen und sind nicht wie beim mikroskopischen Verfahren unwesentlich oder kontrollierbar. Das sehr sperrige Wachstum der Mikrokolonien sowie lysisartige Erscheinungen inaktiver aus-

27 H. Engelhard u. T. Houtermans, Z. Naturforschg. 5 b, 264 [1950].
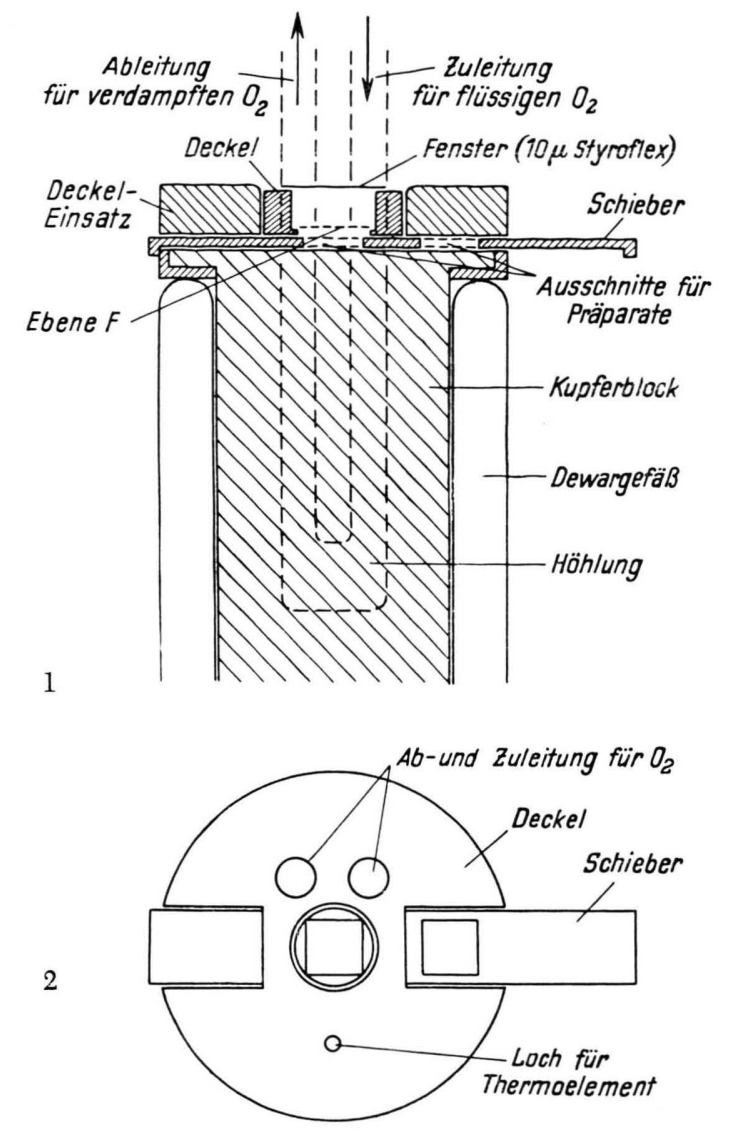

Abb. 1 und 2. Die Versuchsapparatur. Abb. 1 Schnitt. Abb. 2 Aufsicht (Deckeleinsätze entfernt).

geschlüpfter Bazillen sind dagegen Unsicherheitsfaktoren bei der mikroskopischen Zählung. Da die Fehlerquellen beider Methoden jedoch verschieden sind, erschien es zweckmäßig, die Auswertung nach beiden Verfahren durchzuführen.

$$
\begin{aligned}
& \text { Versuchsanordnung für die Bestrah- } \\
& \text { lungmit } \alpha-\text { Teilchen }
\end{aligned}
$$

Als $\alpha$-Strahlenquelle diente wie bei früheren Experimenten auf ein Nickelplättchen niedergeschlagenes Thorium B. Die Aktivität des emanierenden Radiothor-Präparates betrug etwa $25 \mathrm{mg}$ Radiumäquivalent. Die Aktivität des Nickelplättchens, auf dem das Th B bei der Herausnahme annähernd im radioaktiven Gleichgewicht mit dem Radiothor stand, nahm während einer Versuchsdauer entsprechend den Halbwertszeiten des Th B und des Th $\mathrm{C}$ ab, was bei der Berechnung der relativen Dosen berücksichtigt wurde. Innerhalb des hier vorliegenden kleinen Dosisleistungs-Intervalls war keine Abhängigkeit des biologischen Effektes von der Dosisleistung bemerkbar.

28 T. H o u term a n s, Strahlentherapie 92, 423 
Die $\alpha$-Bestrahlungen wurden ebenfalls in der oben beschriebenen und in Abb. 1 und 2 dargestellten Apparatur durchgeführt. Das radioaktive Präparat war in der Ebene F (Abb. 1) fest einmontiert, und die Styroflexfolie wurde durch einen dicken Kunststoff-Pfropfen ersetzt.

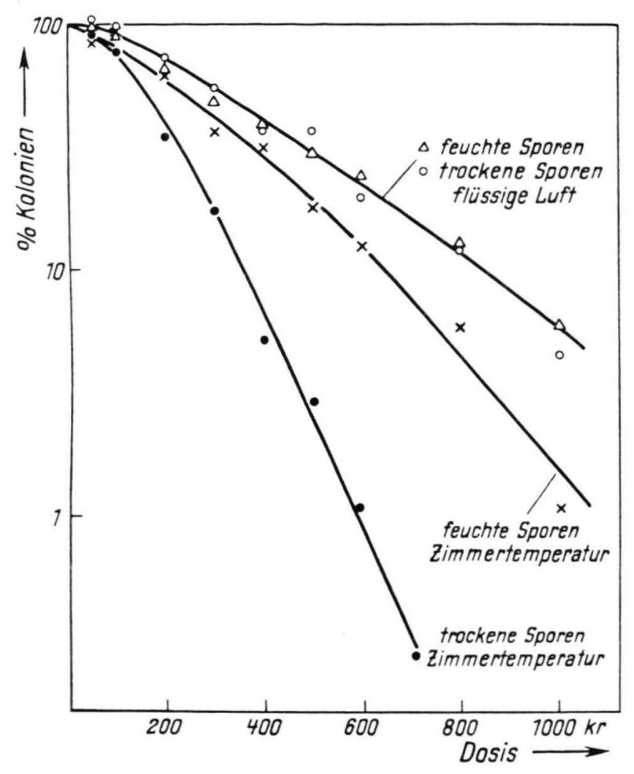

Abb. 3. Dosis-Effekt-Kurven mit 50-kV-Röntgenstrahlen einer Hochleistungsapparatur für trockene und feuchte Sporen von Baz. subtilis.

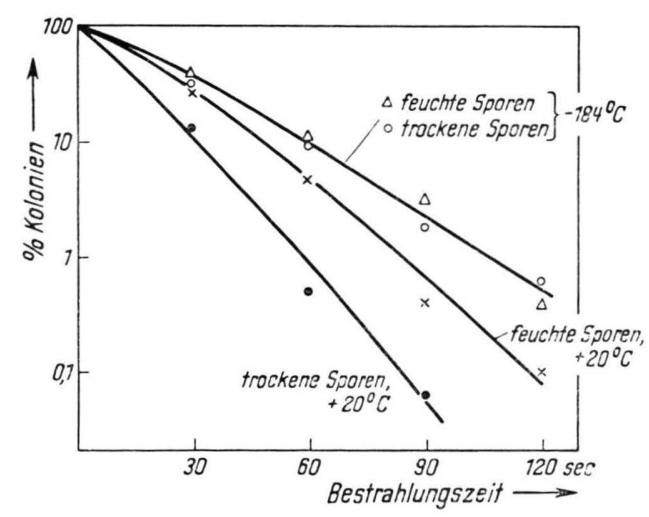

Abb. 4. DEK mit Dermopan-Röntgenstrahlen für Sporen von Baz. subtilis.

\section{Versuchsergebnisse}

In Abb. 3 sind Dosis-Effekt-Kurven feuchter und trockener Sporen dargestellt für die Bestrahlung mit 50-kV-Röntgenstrahlen der Frankfurter Hochleistungsanlage bei Zimmertemperatur und der Temperatur flüssiger Luft. Abb. 4 zeigt die entsprechenden Messungen für die weichere Röntgenstrahlung des Dermopangerätes. Der Einfluß der variierten Parameter auf den Kurvenverlauf ist für beide Strah- lenarten qualitativ gleich. Getrocknete Sporen sind bei Zimmertemperatur empfindlicher als feuchte Sporen. Dieser Effekt scheint für die härtere Strahlung größer zu sein als für die weichere. Dieser Schluß

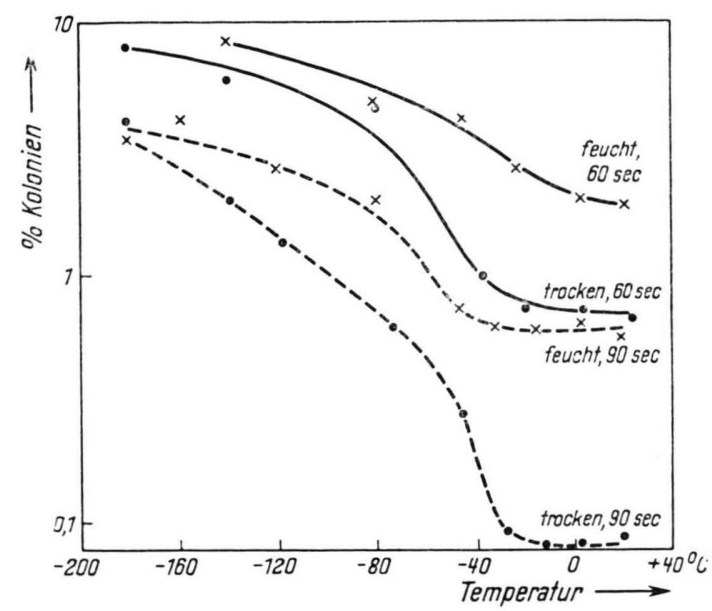

Abb. 5. Inaktivierung von Sporen von Baz. subtilis durch Dermopan-Röntgenstrahlen in Abhängigkeit von der Temperatur.

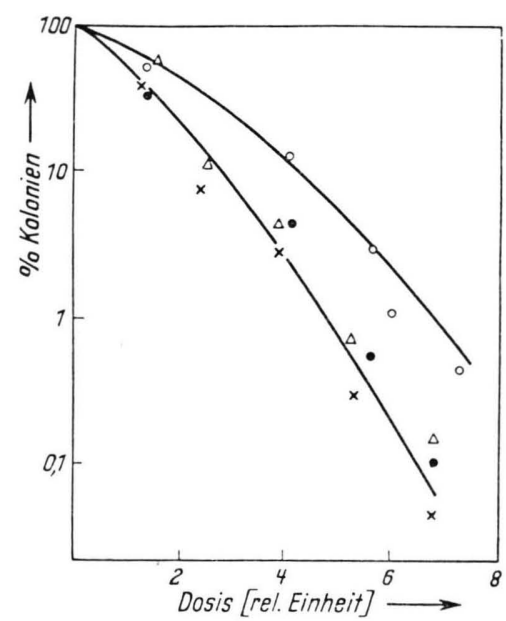

Abb. 6. DEK mit $\alpha$-Strahlen für Sporen von Baz. subtilis. - trockene Sporen, - $184^{\circ} \mathrm{C}$, - trockene Sporen, $+20^{\circ} \mathrm{C}, \triangle \longrightarrow \triangle$ feuchte Sporen, $-184^{\circ} \mathrm{C}, \mathrm{X}-\mathrm{X}$ feuchte Sporen, $+20^{\circ}$ C.

ist jedoch mit einigem Vorbehalt zu ziehen, da die feuchten Sporen bei der härteren Strahlung in Wasser suspendiert, bei der weicheren dagegen auf feuchtem Agar fixiert waren. Die bei der Temperatur flüssiger Luft gemessenen Kurven liegen oberhalb der bei Zimmertemperatur aufgenommenen. Ihr Verlauf ist für trockene und feuchte Sporen praktisch gleich. Gegen die Möglichkeit, daß die bei der härteren Strahlung vorliegende verschiedene geometrische An- 
ordnung für trockene und feuchte Sporen Dosisunterschiede bewirkt haben könnte, sprechen die gleichen Ergebnisse bei tiefer Temperatur.

Für die Dermopanbestrahlung wurden Versuche durchgeführt, bei denen die Temperatur kontinuierlich variiert wurde. Abb. 5 stellt Überlebenden-Prozentsätze bei konstanter Dosis in Abhängigkeit von der Temperatur dar. Es wurde darauf verzichtet, die Temperaturabhängigkeit allgemeiner zu formulieren, etwa durch Angabe der Änderung der Letaldosis, da die Genauigkeit der nicht exponentiellen DEK nicht ausreicht, um die Annahme eines einfachen Dosis-Reduktionsfaktors zu rechtfertigen.

Beim Übergang zu tiefen Temperaturen findet zunächst keine Empfindlichkeitsänderung statt. Erst unterhalb von $-30^{\circ} \mathrm{C}$ erfolgt eine merkliche Empfindlichkeitsabnahme. Die Kurven für feuchte und trokkene Sporen nähern sich einander, und bei sehr tiefen Temperaturen sind die Inaktivierungs-Wahrscheinlichkeiten praktisch gleich.

Die Versuchsergebnisse für $\alpha$-Bestrahlung sind in Abb. 6 und 7 dargestellt. Abb. 6 zeigt DEK für $+20^{\circ} \mathrm{C}$ und $-184^{\circ} \mathrm{C}$, Abb. 7 die ÚberlebendenProzentsätze als Funktion der Temperatur mit der Dosis als Parameter. Die DEK für feuchte Sporen und die der trockenen Sporen bei $+20^{\circ} \mathrm{C}$ verlaufen nicht deutlich unterschiedlich. Möglicherweise sind dic feuchten Sporen bei $+20^{\circ} \mathrm{C}$ etwas empfindlicher. Nur die Kurve für trockene Sporen bei $-184^{\circ} \mathrm{C}$ liegt deutlich oberhalb der anderen. Für trockene Sporen zeigen die Temperaturkurven einen ähnlichen Verlauf wie für Röntgenstrahlen. Bei den feuchten Sporen dagegen hat die Temperatur keinen deutlichen Einfluß. Nach den in Abb. 7 wiedergegebenen Punktfolgen scheint ein sehr flaches Minimum bei mittleren Temperaturen möglich; dieses ist jedoch nicht genügend gesichert. Außerdem ist die relative Lage der Kurven für feuchte und trockene Sporen anders als bei Röntgenstrahlen, da die Kurve für trockene oberhalb der Kurve für nasse Sporen verläuft, während es für Röntgenstrahlen umgekehrt ist.

\section{Diskussion der Versuchsergebnịsse}

Der Befund, daß feuchte Sporen bei Zimmertemperatur weniger empfindlich sind als trockene Sporen, mag zunächst der Erwartung widersprechen, die bestenfalls eine Unabhängigkeit des Effektes vom Wassergehalt zulassen möchte. Die Annahmen, die einer solchen spontanen Erwartung zugrunde liegen, sind offensichtlich revisionsbedürftig und sollen zu- nächst einer näheren Untersuchung unterzogen werden:

Man setzt voraus, daß die in der trockenen Substanz ablaufenden Reaktionsketten, die zum beobachteten Effekt beitragen, sich auch bei Anwesenheit von Wasser unbehindert ausbilden. Das bedeutet:

1. Die in der nicht wäßrigen Substanz erfolgenden primären Absorptionsprozesse sind, soweit sie für den Effekt von Bedeutung sind, im trockenen und feuchten System identisch.

2. Die sich in der trockenen Substanz an diese Primärabsorptionen anschließenden Folgereaktionen laufen auch bei Anwesenheit von Wassermolekülen ungehindert $a b$.

3. Ausgehend von der gut fundierten Erfahrung, daß die bei der Bestrahlung von Wasser absorbierte Energie zum Teil zur Erzeugung sehr reaktionsfähi-

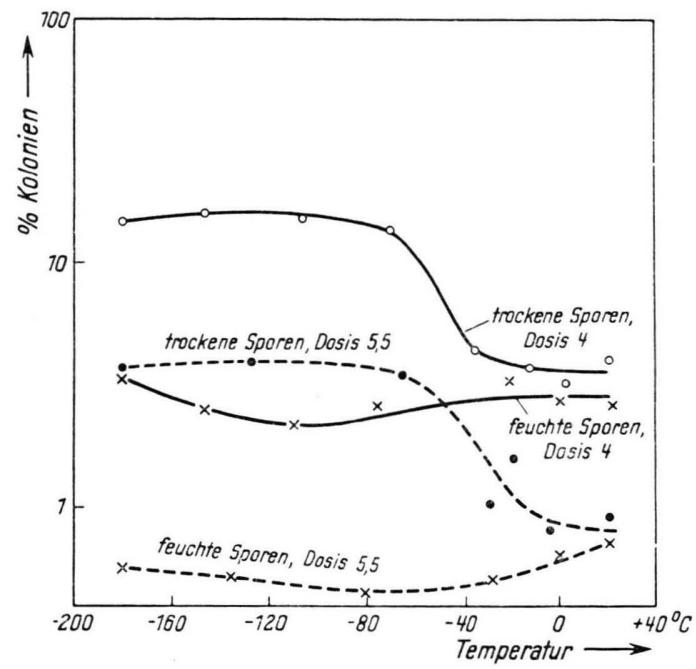

Abb. 7. Inaktivierung von Sporen von Baz. subtilis durch $\alpha$-Strahlen in Abhängigkeit von der Temperatur.

ger Radikale und deren Folgeprodukten dient, wird weiterhin angenommen, daß die bei Anwesenheit von Wasser zusätzlich in diesem absorbierte Energie Reaktionsketten auslöst, die zum beobachteten Effekt beitragen, jedoch nicht mit den auch im trockenen System ablaufenden Reaktionen interferieren können. Es werden also zwei säuberlich voneinander getrennte Reaktionsgruppen vorausgesetzt.

Dies sind Simplifikationen, über deren ZweckmäBigkeit nur Experimente entscheiden können, denn:

1. Entgegen der Annahme 1 wäre es möglich, daß bereits die in der organischen Substanz erfolgenden Primärprozesse durch Wasser beeinflußt werden. Adsorptionsprozesse können zu neuen Energieniveaus 
führen. Das zeigen $\mathrm{Ewles}$ und $\mathrm{F}$ arnell ${ }^{29}$ in Versuchen über die Lumineszenz feuchter und trokkener Stoffe. Energieniveau-Änderungen können jedoch für die Primärverteilung der Energie von Bedeutung sein.

2. Die durch die Wasseraufnahme bedingten strukturellen Veränderungen könnten eine Änderung der sich an die Primärreaktion anschließenden Folgereaktionen bewirken.

3. Der spekulative Charakter der Annahme 3 ist wohl ohne weiteres evident.

Läßt man eine der obigen Einschränkungen fallen, so erscheinen die Möglichkeiten, daß Wasseraufnahme zu einer Erhöhung, Erniedrigung oder Nicht-Beeinflussung des Effektes führt, primär gleichberechtigt. Sowohl eine durch Wasser bedingte Schutzwirkung wie auch eine nicht monotone Funktion für die $\mathrm{Ab}$ hängigkeit vom Feuchtigkeitsgrad (E h r e n b e r g ${ }^{19}$ ) sind ohne weiteres plausibel.

E hre n berg diskutiert seine Befunde bei Samen und Stärke offenbar im Sinne der hier unter 2 aufgeführten Erklärungsmöglichkeit, wenn er annimmt, daß ein Teil der primär im organischen Material absorbierten Energie über das Wasser abgeleitet und zu Reaktionen verwandt wird, die im trockenen $\mathrm{Zu}$ stand nicht stattfinden.

Versuche einer Deutung des Temperatureinflusses auf biologische Objekte erscheinen auf Grund der eingangs erwähnten, bereits für trockene und reine Substanzen komplexen Befunde nicht sehr aussichtsreich. Falls eine Unstetigkeit beim Phasenwechsel vorliegt, kann man wenigstens auf eine wesentliche Beteiligung von Diffusionsvorgängen von Substanzen schließen, deren Lebensdauer auch in gefrorenem Milieu kleiner ist als die Zeit von ihrer Entstehung bis zur Verflüssigung des Milieus. Daß Temperatur-Erniedrigung immer eine Herabsetzung des Effektes bewirkt, ist nicht nur durch Experimente widerlegt, sondern auch auf Grund theoretischer Überlegungen nicht unbedingt zu erwarten. In einem System, das aus mehreren Substanzen besteht, kann für den beobachteten Effekt eine Kombination von sich günstig und ungünstig auswirkenden Reaktionsabläufen verschiedener Temperaturabhängigkeit maßgeblich sein. Daraus können durchaus Temperaturfunktionen resultieren, die Maxima und Minima aufweisen, wie dies z. B. bei der Zerstörung von Behensäure durch $\alpha$-Teilchen der Fall ist ${ }^{30}$; das Behensäure-Molekül

29 J. Ew les u. G. C. F a rne 11, Proc. physic. Soc., Sect. A 62, 216 [1949].

30 W. L. Whit eh ead, G. Goodman u. I. A. B r e g e r, J. Chim. physique 48, 184 [1951]. kann selbst als System aufgefaßt werden. Als Beispiel für eine solche Kombination verschieden temperaturabhängiger Reaktionen führt B e rgster $\mathrm{m}$ a n $\mathrm{n}^{31}$ seine Ergebnisse über die durch Röntgenstrahlen induzierte Chemolumineszenz von Luminol in wäßrigen Lösungen an. Er berichtet, daß sich die Lumineszenz für das System Wasser-Luminol beim Einfrieren nicht ändert und die Zugabe von Cystein die Leuchterscheinungen im flüssigen, nicht dagegen im gefrorenen Zustand unterdrückt. In dem System Wasser-Luminol-Cystein würde also durch Temperatur-Erniedrigung der beobachtete Effekt dadurch erhöht, daß allein die Schutzreaktion temperaturabhängig ist.

$\mathrm{Zu}$ den in dieser Arbeit beschriebenen Versuchsergebnissen läßt sich folgendes sagen:

Bei der Inaktivierung von Sporen durch $a$ - und Röntgenstrahlen scheinen Diffusionsprozesse von kurzlebigen Substanzen keine wesentliche Rolle zu spielen, da eine Änderung des Aggregatzustandes den Effekt nicht beeinflußt. Bezeichnet man die die Manifestierung des Effektes begünstigenden Reaktionen als schädigend, alle anderen dagegen als schützend, so folgt aus der für trockene Sporen gemessenen Temperatur-Abhängigkeit, daß sich mit sinkender Temperatur die Verteilung der absorbierten Energie zugunsten der Schutzreaktionen verschiebt. Da der Funktionsverlauf für $\alpha$ - und Röntgenstrahlen sehr ähnlich ist, erscheint die Annahme, daß hier die zur Inaktivierung führenden Reaktionsabläufe temperaturempfindliche Prozesse einschließen, die für beide Strahlenarten identisch sind, diskutabel.

Bei Röntgenstrahlen wirkt sich die Anwesenheit von Wasser als Vermehrung der Schutzreaktionen aus. Diese zusätzlichen Schutzreaktionen schließen temperaturabhängige Prozesse ein, die mit sinkender Temperatur abnehmen. Gegen $\alpha$-Strahlen dagegen wirkt Wasser nicht schützend, sondern die Schutzreaktionen werden behindert. Die zur Behinderung führenden Reaktionsabläufe schließen temperaturabhängige Prozesse ein, die mit sinkender Temperatur abnehmen.

Eine solche Diskussion ist natürlich nichts anderes als eine Beschreibung der Versuchsergebnisse in einer speziellen Terminologie. An sich erhofft man dagegen Aufschlüsse, die über die gemessenen Tatsachen selbst hinausgehen; das heißt, man möchte aus den vorliegenden experimentellen Befunden und anderen bekannten Phänomenen und gesicherten Gesetzmä-

31 H. B e r g s t e rm a n n, Naturwissenschaften 42, 152 [1955]. 
Bigkeiten unter Zuhilfenahme von Hypothesen ein System konstruieren, dessen Glieder in widerspruchsfreiem kausalem oder logischem Zusammenhang stehen. Der Wert einer solchen Konstruktion ist, jedenfalls in einer Wissenschaft, die das Prädikat „empirisch" für sich in Anspruch nimmt, durch die Relation der „faktischen“ zu den hypothetischen Elementen gegeben. Obwohl es leicht ist, für den vorliegenden Fall eine ganze Reihe von möglichen Systemen aufzuzeigen, findet die Verfasserin dieser Arbeit jedenfalls keines, in dem die eingebauten Fakten die hypothetischen Annahmen überwiegen.

Endlich sollen noch die Versuche von Proctor u. a. ${ }^{20}$ über die Inaktivierung von Sporen von $\mathrm{Baz}$. subtilis durch 200-kV-Kathodenstrahlen erwähnt werden. Die Verfasser fanden auf Grund einer statistischen Auswertung zahlreicher Einzelexperimente für kälte-getrocknete Sporen eine um 10\% niedrigere Letaldosis als für die in Salzlösung aufgeschwemmten Sporen, sowie für in Salzlösung aufgeschwemmte Sporen bei $-78^{\circ} \mathrm{C}$ eine um $10 \%$ höhere Letaldosis als bei $-2^{\circ}$ C. Diese Daten stimmen im Rahmen der Meßgenauigkeit mit den eigenen Befunden für $\alpha$ Strahlen, nicht dagegen mit denen für Röntgenstrah- len überein, während man das Gegenteil vermuten würde. Selbstverständlich waren weder Art und Züchtung der Sporen, noch die Versuchsbedingungen völlig gleich. Weder die Annahme, daß derartige Unterschiede sich maßgeblich auf die Versuchsergebnisse auswirken, noch die Alternative, daß tatsächlich die Strahlenwirkung der $\alpha$ - und Kathodenstrahlen ähnlich, die der Röntgenstrahlen dagegen grundsätzlich von beiden verschieden ist, ermutigt jedoch bei dem gegenwärtigen Stand unserer Kenntnisse zu einer speziellen Deutung des Wirkungsmechanismus.

Für die Ermöglichung der Bestrahlungen an der Hochleistungs-Röntgenanlage des Max-Planck-Institutes für Biophysik in Frankfurt a. M. und für das meiner Arbeit entgegengebrachte Interesse möchte ich Herrn Prof. Dr. B. R a j e w s ky sowie seinen Mitarbeitern, die mich bei der Ausführung der Versuche unterstützt haben, hier noch einmal herzlich danken.

Ferner danke ich Herrn Prof. Dr. F l a m me r s f eld vom 2. Physikalischen Institut der Universität Göttingen für die Überlassung des $\alpha$-Präparates und Herrn Prof. Dr. H. E $\mathrm{n}$ g e $1 \mathrm{~h}$ a r d vom Institut für physiologische Chemie der Universität Göttingen für die Überlassung des Dermopan-Röntgengerätes.

Die Arbeit wurde durch Unterstützung der Deutschen Forschungsgemeinschaft ermöglicht.

\title{
Vergleich der Inaktivierungen bei Escherichia coli $B$ durch organische Peroxyde und UV-Bestrahlung unter besonderer Berücksichtigung der Wärme- und Photo-Reaktivierbarkeit
}

\author{
Von Werner Stein und Wolfgang Laskowski
}

Aus dem I. Physikalischen Institut der Freien Universität Berlin und dem Institut für Genetik der Freien Universität Berlin

(Z. Naturforschg. 11 b, 643-653 [1956]; eingegangen am 28. April 1956)

\begin{abstract}
Inactivation of E. coli strain $\mathrm{B}$ by $\mathrm{H}_{2} \mathrm{O}_{2}$ treated broth has been studied in relation to peroxide concentration and time of contact. The rate of survivals was found to depend on the product of these two values. Below a certain limit of this product there occurred a reactivation by incubation at higher temperatures that showed a $D R F$ value of (3-4): 1; after UV irradiation this value is $(10-20): 1$. There was also found a reactivation of peroxide inactivated cells by irradiation with wave lengths $>312 \mathrm{~m} \mu$. The average $D R F$ value obtained was $1,3: 1$ whereas after UV irradiation this value was about $4: 1$. Although the greater part of peroxide inactivation is connected with irreversible processes probably in the cytoplasm the conclusion is drawn that the reversible part of peroxide inactivation proves the idea that UV inactivation is related to a secondary production of poisons similar to organic peroxides.
\end{abstract}

$\mathrm{I}$

n einer früheren Arbeit ${ }^{1}$ war im Anschluß an eine Modellvorstellung über die Folgeprozesse von UVBestrahlung die Vermutung ausgesprochen worden, daß eine Inaktivierung der Coli-Zelle mit organischen

1 W. S tein u. W. Harm, Z. Naturforschg. 8 b, 742 [1953].
Peroxyden auch photo-reaktivierbar sein könnte. Im gleichen Zusammenhang wurde darauf hingewiesen, daß anderenfalls die vermutete sekundäre Peroxydbildung nach einer UV-Bestrahlung zeitlich nach dem photo-reaktivierbaren Stadium anzusetzen sei, sofern man an den Grundvorstellungen dieses Modells fest- 